\title{
AMERIGO DI NARBONA, ULTIMO SOVRANO DI ARBOREA?
}

\author{
LUCIANO GALLINARI \\ Istituto sui Rapporti Italo-Iberici \\ (CNR, Cagliari. Italia)
}

In tutta la storiografia che fino ad ora si è occupata del lungo conflitto che durante la metà del Trecento e i primi vent'anni del Quattrocento ha opposto il Regno di "Sardegna e Corsica" al Regno o "Giudicato" di Arborea, e che ha trattato dell'ultima fase di esistenza dello Stato arborense, è affermato che l'ultimo sovrano oristanese fu il visconte di Narbona, Guglielmo II, discendente della dinastia sovrana sarda per parte della nonna Beatrice, figlia del sovrano Mariano IV, chiamato nel 1408 dai Sardi a ricoprire il trono vacante dopo la morte di Mariano V avvenuta l'anno precedente!

'Sul legame dinastico che univa Guglielmo II di Narbona alla Casa dei Bas-Serra, giudici di Arborea, vedasi Genealogie medioevali di Sardegna, a cura di L.L. BrooK; F.C. CaSULA; M.M. Costa; A.M. Oliva; R. Pavoni; M. Tangheroni, Cagliari-Sassari, 1984, Lemma e Tavola XXXVII, 26, pp. 146-147 e 438, in cui il sovrano oristanese è però indicato col numerale III. In merito a ciò ci siamo serviti degli studi condotti dalla studiosa francese Jacqueline Caille, massima conoscitrice delle vicende storiche e dinastiche dei signori di Narbona, che attribuisce invece al sovrano arborense il numerale II. Cfr. J. CAILLE, La famiglia dei visconti di Narbonne nella storia della loro città, in La Sardegna giudicale e il medioevo europeo. Secondo incontro di storia medioevale comparata Oristano e Narbonne (Oristano 11-14 ottobre 1996), a cura di M. G. Tarantini e L. Gallinari, attualmente in corso di stampa.

Sugli ultimi anni di vita e sugli avvenimenti che portarono alla morte del giudice d'Arborea Mariano V, ultimo esponente della dinastia Bas-Serra, vedasi invece L. GallinaRI, Nuovi dati su Mariano V sovrano di Arborea, in "Medioevo. Saggi e rassegne”, 21 (1996), pp. 127-146. 
Questa ricostruzione degli avvenimenti appare messa in discussione -almeno ad un livello formale - da quanto riportato dalla fonte che ci accingiamo a pubblicare.

Non desiderando riproporre in questa sede avvenimenti e personaggi sui quali ci siamo già soffermati in altri nostri lavori, ci limitiamo a ricordare che dopo essere stato giurato giudice di Arborea nel gennaio del 1409, Guglielmo II di Narbona guidò gli eserciti giudicali nella battaglia di Sanluri del 30 giugno dello stesso anno, conclusasi con una pesantissima sconfitta per i Sardi. Dopo aver tentato per diversi anni di mantenere i proprî possedimenti sardi - anche dopo la secessione di un terzo del giudicato passato all'obbedienza regia nel marzo del 1410 sotto la guida di Leonardo Cubello, nominato marchese di Oristano- il visconte di Narbona raggiunse nel 1414 un accordo definitivo con il sovrano aragonese Ferdinando I de Antequera, per la vendita dei diritti sovrani che vantava sul regno di Arborea.

In realtà, l'intricata vicenda dell'acquisto delle prerogative che Guglielmo II aveva sullo Stato oristanese non si risolse subito. Al contrario, occorsero altri sei anni di estenuanti trattative tra i procuratori del visconte e quelli di Ferdinando I prima e di Alfonso V poi perchè il 17 agosto 1420 i diritti sul plurisecolare regno arborense passassero dalle mani del francese a quelle sovano aragonese ${ }^{2}$.

All'interno dunque di questo periodo di tempo - sei anni, dal 1414 al 1420 - va situato l'interessantissimo documento che presentiamo in questo nostro lavoro. Per essere più precisi, dall'esame di alcune parti del testo stesso - mancante purtroppo dell'indicazione dell'anno- riteniamo che esso sia da riferirsi al luglio 1414 allorché si concretò in via definitiva -almeno sul piano formale- la vendita dei diritti del visconte di Narbona sul Giudicato d'Arborea.

Nel dettaglio, si tratta di una lunga relazione, purtroppo anonima, inoltrata da un ufficiale regio al sovrano argonese - dovrebbe trattarsi di Ferdinando I - che racconta in maniera estremamente dettagliata quanto

\footnotetext{
2Per gli accordi raggiunti tra Ferdinando I e Guglielmo II di Narbona sulla vendita delle prerogative di quest'ultimo sul Giudicato di Arborea vedasi almeno F. C. CASULA, La Sardegna aragonese. 2 La Nazione Sarda, Sassari, 1990, pp. 585-590. Sulle complesse modalità di pagamento messe in atto dai sovrani aragonesi vedasi invece P. ROQUÉ, I fiorini, il re e il visconte di Narbona, in "Quaderni sardi di storia”, 3 (luglio 1981-giugno 1983), pp. 50-78.
} 
accaduto nei primi mesi dell'estate 1414 nella città di Sassari, divenuta capitale del regno giudicale d'Arborea tra il 1410 e il 1420 .

Abbiamo circoscritto al 1414 la datazione del documento basandoci sul tenore degli avvenimenti narrati e sui protagonisti. In particolar modo, ciò che ci ha fatto propendere per questa ipotesi è l'atmosfera di stupore e incredulità sia dei Sassaresi che degli stessi Francesi presenti nella capitale giudicale dinanzi al diffondersi della notizia che il visconte di Narbona aveva raggiunto un accordo con il sovrano aragonese, che prevedeva il passaggio a quest'ultimo di tutti i possedimenti e le prerogative che Guglielmo II vantava in Sardegna.

Oltre a questa atmosfera di estrema incertezza nei confronti di un futuro minaccioso sempre più incombente, sembra leggersi fra le righe della nostra fonte anche un'altra eco della crisi ormai irreversibile del rapporto di fiducia tra il popolo giudicale, ancora fedele alla causa nazionalista, e il giudice Guglielmo II. Una sfiducia che sembrò non investire al momento i rappresentanti del visconte - cioè il fratello Amerigo di Narbona e il suo tutore Pere de Montbrun signore di Maurellas, veri protagonisti della nostra fonte - né la figura del giudice in quanto capo dello Stato, il carisma e la forza agglutinatrice del quale rimasero inalterati fino al termine dell'esistenza del regno arborense ${ }^{3}$.

Come abbiamo già detto, il documento fu redatto con ogni probabilità da un ufficiale regio della Corona d'Aragona che ricavò notizie di primissima mano sulla situazione interna a Sassari. A fornirle a lui e al governatore generale di Catalogna fu "un gentil hom castellà" chiamato Rodrigo de San Juan. Costui, infatti, pur essendo "aquell qui tractava ab vostra senyoria e ab los Sarts", continuò a fingersi un fedele seguace del visconte di Narbona nella capitale giudicale ${ }^{4}$. Grazie a ciò riuscì a venire a

${ }^{3}$ F.C. Casula, La Storia di Sardegna, Pisa-Sassari, 1992, p. 438 sottolinea come nonostante che Leonardo de Alagón, ultimo marchese di Oristano, discendente della famiglia dei giudici di Arborea, fosse insorto nel 1470 contro il viceré del Regno di "Sardegna e Corsica", Nicolò Carròs, gli Oristanesi insorsero al suo fianco al grido di "Arborea, Arborea". Dinanzi al rischio potenziale che tutta l'isola potesse insorgere dietro il richiamo nazionalista - ventilato dal viceré al sovrano aragonese- Giovanni II condannò a morte l'Alagón e i suoi seguaci, sequestrando loro tutti $i$ beni in favore del patrimonio regio e destinando così ad una cruenta estinzione il Casato dei Marchesi di Oristano. Su Leonardo de Alagón e l'epilogo della sua insurrezione vedasi M. SCARPA SENES, La guerra e la disfatta del marchese di Oristano, dal manoscritto di Giovanni Proto Arca, Cagliari, 1997.

${ }^{4}$ Pur nella laconicità del testo a questo proposito, emerge un altro elemento su cui fermarsi a riflettere. Da quanto affermato dall'ufficiale estensore del nostro documento, sembrerebbe potersi evincere che il succitato Rodrigo de San Juan non si limitasse a passare le informazioni 
conoscenza delle informazioni di seguito riportate nella presente lettera, le quali gli furono rivelate da un francese del seguito di Guglielmo II, che faceva rientro a Narbona per recarsi alla presenza del giudice dietro incarico del fratello Amerigo e del Maurellas. Il suddetto francese ritenendo che il San Juan "qui solia estar ab lo vescomte ... encara hy stigués", gli riferì tutte le informazioni che questi a sua volta raccontò al governatore di Catalogna e allo scrivente, il quale si affrettò ad informarne il sovrano affinché potesse prendere i provvedimenti necessarî al caso.

Le notizie riferite erano estremamente interessanti per il monarca iberico. Infatti, in base ad esse risultò che Barisone Cano, cittadino sassarese fedele alla causa aragonese che si era visto sequestrare tutti i suoi beni dal "giudice" d'Arborea Guglielmo II, si era recato a Alghero in compagnia del conte di Quirra, Berenguer Carròs, governatore generale del Regno di "Sardegna e Corsica". Dopo di ciò, i cittadini di Sassari avevano preso a rumoreggiare con sempre maggiore forza ${ }^{5}$. La situazione dovette apparire

sulla situazione all'interno del giudicato al re aragonese, ma trattasse nel contempo anche con i Sardi. Non risulta del tutto chiaro se in questo caso si debba intendere che egli passasse notizie sugli spostamenti catalano-aragonesi ai Sardi giudicali, o piuttosto che fornisse informazioni sia sugli iberici che sul visconte di Narbona agli isolani. Sembrerebbe da preferirsi questa seconda ipotesi.

${ }^{5}$ Pochi giorni dopo il documento da noi esaminato, il 23 luglio dello stesso anno, il re aragonese scrisse a Guglielmo II di Narbona di aver saputo personalmente da Barisone Cano presente a Saragozza, che essendosi questi recato a Alghero contro la volontà del giudice d'Arborea - della qual cosa il sovrano lo aveva molto rimproverato- Amerigo di Narbona e il signore di Maurellas avevano fatto arrestare suo figlio, e avevano fatto cacciare di casa la moglie e 7 altri figli impadronendosi di tutti i loro beni. Atteggiamento questo non gradito a Ferdinando I, che ordino al visconte di ordinare a sua volta al fratello di liberare il fíglio del Cano prigioniero e di rimettere la madre di questi in possesso di ogni bene spettantele. ACA, C, reg. 2388, ff. $5 \mathrm{v}-6 \mathrm{r}$. Circa un mese dopo la stesura della nostra fonte, in data 9 agosto 1414 , il re Ferdinando I scrisse al suo procuratore generale del Regno di "Sardegna e Corsica", Guillem Satria, ordinandogli di rifornire di denaro a sufficienza sia il già citato Barisone Cano che suo genero, Antonio Marongio, i quali per motivi non sconosciuti all'ufficiale regio non potevano far rientro a Sassari, ove tutti i loro beni erano stati sequestrati da Amerigo di Narbona. ACA, C, reg. 2386, f. 29r. La difficile situazione del Cano non dovette risolversi in breve tempo nonostante l'intervento regio, dal momento che alcuni mesi più tardi, il 4 dicembre 1414, Ferdinando I in una sua missiva a Guglielmo II era costretto a ritornare sull'argomento, facendo presente al suo destinatario che niente di quanto richiestogli a proposito dei familiari e dei beni del Cano era stato messo in pratica. Pertanto il sovrano tornava a sollecitare il visconte di Narbona perché si attivasse per rendere giustizia. ACA, C, reg. 2389, f. 38r-v. Neppure questo sollecito regio sortì alcun effetto: il 24 gennaio 1416 Ferdinando I, in considerazione del fatto che il figlio del Cano, Bartolomeo, continuava ad essere prigioniero del visconte e che $\mathrm{i}$ beni sequestrati alla famiglia non erano ancora stati restituiti, informò Guglielmo II di aver comunicato a Joan Tavesca, segretario dell'infante Alfonso, le sue decisioni in merito a tale problema, pregandolo nel contempo di scrivere al fratello Amerigo e al suo procuratore di liberare il suddetto prigioniero. ACA, C, reg. 2389, f. 134r. 
seria se Amerigo di Narbona, che reggeva lo Stato giudicale per il fratello, con tutto il suo seguito di francesi decise di rifugiarsi dentro il castello di Sassari. Lì, sempre secondo la nostra fonte, i francesi "han estat III jorns que no son exits del castell". A causare questa tensione improvvisa tra Sardi e Francesi fu lo spargersi della notizia che il visconte di Narbona aveva venduto i suoi diritti sul "Giudicato" di Arborea al re d'Aragona.

È questo il primo degli elementi che ci ha fatto propendere per datare il nostro documento al 1414. Infatti, non avrebbe avuto senso una simile reazione di stupore e indignazione da parte dei Sassaresi se questa non fosse stata la prima volta che essi sentivano parlare della cessione dei diritti regî di Guglielmo II al re d'Aragona. Decisione che comportava l'inevitabile ritorno alla dominazione iberica, da loro particolarmente avversata, dopo la cacciata dei Catalano-Aragonesi dalla città effettuata da Brancaleone Doria il 21 agosto $1391^{6}$.

Già altre volte e da più parti è stata sottolineata la fermezza con cui i cittadini di Sassari cercarono di opporsi ad un epilogo invitabile. Anche nella fonte che presentiamo in questo lavoro si coglie molto bene questo spirito di indipendenza - "ells no eren cans perquè deguessen ésser venits" - , e la disponibilità a portare questo atteggiamento fino alle estreme conseguenze: "abans [di sottomettersi al dominio catalano-aragonese] se mengerien los fills que no consentrien que fossen de la casa d'Aragó"?

Vista la pericolosità della situazione decise, di intervenire il Maurellas, il quale si affrettò a calmare gli animi dei Sassaresi sostenendo

${ }^{6}$ F. C. Casula, La Sardegna aragonese. 2, cit., pp. 454-455.

${ }^{7}$ Riteniamo perfettamente paradigmatica di questo stato d'animo di profonda avversione provato dai Sardi, e dai Sassaresi in particolare, la lettera inviata il 6 gennaio 1392 dal podestà di Sassari, Pietro de Marongio, dai consiglieri e probiuomini della città al governatore del Capo di Logudoro, residente a Alghero, in merito alla restituzione al re d'Aragona della città e delle terre recuperate da Brancaleone Doria nel Nord dell'isola a seguito della sua fortunata campagna militare del 1391. In essa il Marongio ribadì a chiare lettere che "Sasser es fundada per Sarts propris e naturals, e no pas per Cathalans ni per altra generacio", e che "en espay del XL anys los dits Sarts ne son estats axi gitats de fora anant dispersos e mendicants, e s.eren mesos en la dita ciutat $\mathrm{M}$ heretats de Cathalans en les possecions e bens dels dits mesquins Sarts siutadans e edificadors de la dita ciutat". Non pago di ciò, il podestà aggiunse anche che i Sardi "son serts que.ll rey no tramet en la illa si no menyadors e robadors, per so que viscan de robaria, perque a vos torna en gran plaer que les robaries se fassen en la illa, per tal que puscats ben viure e grassament, e per so que en Cathaluya no podets viure, venits en la illa per robar e menyar ..." Cfr. F. C. Casula, Carte Reali Diplomatiche di Giovanni I il Cacciatore, re d'Aragona, riguardanti l'Italia, Padova, 1977, doc. 34, pp. 61-63. 
che era impensabile che il signore di Arborea si fosse accordato con il sovrano aragonese per vendergli tutti i diritti che vantava in Sardegna ${ }^{8}$.

Questo è il secondo elemento che ci ha spinto a proporre la data del 1414 per la nostra fonte. In questo frangente il Maurellas - non sappiamo quanto in buona fede e quanto invece desideroso di scampare da un pericolo di morte che pareva sempre più imminente- sembrò escludere categoricamente anche solo la possibilità che Guglielmo II avesse deciso di privarsi dei proprî possedimenti sardi. A conti fatti, crediamo più probabile che sia lui che il fratello del visconte fossero stati colti alla sprovvista dalle voci che iniziavano a circolare a Sassari, dal momento che la conferma della fondatezza di tali voci arrivò nella capitale giudicale solo poco dopo ${ }^{9}$.

Tutto ciò ciò induce a fare anche un'altra triplice riflessione in merito a questa vicenda. O la decisione di Guglielmo II di vendere le proprie prerogative giudicali fu presa tanto repentinamente da non avere il tempo materiale di avvisare il proprio fratello e il suo tutore; o il visconte di Narbona non ritenne opportuno avvisarli di quanto già deciso; oppure tutto era stato già concordato fra i tre, e sia Amerigo di Narbona che il signore di Maurellas presero a recitare una sorta di commedia. Nei primi due casi Guglielmo II avrebbe esposto il fratello e il tutore a serissimi rischi di incolumità personale; nel terzo, invece, il rischio sarebbe stato per così dire più calcolato. La successiva evoluzione degli avvenimenti indurrebbe a pensare che Guglielmo II non abbia informato il fratello Amerigo e il signore di Maurellas delle sue intenzioni.

A prescindere da ciò, dopo aver udito quanto detto dal signore di Montbrun, i Sassaresi parvero riguadagnare la calma.

Tuttavia la situazione era destinata a riprecipitare nel giro di pochissimo tempo, e questa volta in maniera anche più grave di prima. A

\footnotetext{
${ }^{8}$ Per essere esatti, nel testo compare che il signore di Maurellas "los preychà". Il verbo "predicar" (qui nella sua forma medioevale "preicar, o preycar") quando è seguito dal complemento diretto di colui al quale ci si rivolge significa annunciare una cosa come autentica e come norma da seguire. Cfr. Diccionari Catalì-Valencià-Balear, a cura di A. M ${ }^{\mathrm{a}}$. AlcoverF. de B. MOLL, tomo VIII, voce "Predicar", pp. 822-823. Questo potrebbe spingere ad ipotizzare che effettivamente il signore di Maurellas non fosse al corrente di quanto appena deciso dal visconte di Narbona.

${ }^{9} \mathrm{Le}$ voci a cui si fa riferimento nel nostro documento furono alimentate dal fatto che il 2 giugno 1414 il re d'Aragona Ferdinando I scrisse una lettera al podestà, ai consiglieri e ai probiuomini di Sassari per informarli che a seguito degli accordi raggiunti con il visconte di Narbona, la città di Sassari e le sue appendici e il restante territorio del "Giudicato" di Arborea entro breve tempo sarebbero dovuti tornare all'obbedienza regia. Cfr. L. D'ARIENzo, Documenti sui visconti di Narbona e la Sardegna, Padova, 1977, vol. I, doc. 28, p. 16.
} 
originare i nuovi disordini fu l'arrivo a Sassari da Saragozza di uno scudiero del visconte di Narbona, Roger, proprio in concomitanza con questi sommovimenti. Non appena egli raccontò che Guglielmo II e Ferdinando I d'Aragona avevano raggiunto l'accordo, come riporta la nostra fonte "si la primera remor fonch gran, molt major fonch aquesta altra".

Allora però i Sardi non si limitarono a rumoreggiare, ma presero la decisione di rivolgersi direttamente a Amerigo, che faceva le veci del fratello. Una volta ancora non possiamo esimerci dal notare come traspaia chiaramente dal testo della nostra fonte l'estremo tentativo dei Sassaresi e dei Sardi giudicali di preservare la propria indipendenza. Fu detto, infatti, in maniera esplicita a Amerigo di Narbona e al signore di Maurellas che se fossero stati disposti a sottostare agli ordini dei Sassaresi, questi avrebbero speso e impegnato nella lotta contro gli Iberici sia le proprie persone che $i$ proprî beni ("a n'Aymerich de Narbona que si ell volia estar a regiment dels de Sàsser e que fessen ço que ells volguessen, que ells dependrien bens e personas e quant haven").

In caso di rifiuto, essi dichiararono in maniera chiara che la loro intenzione era di continuare lo scontro con la forze regie facendo però a meno dell'appoggio francese.

Questo atteggiamento verso la famiglia dei visconti di Narbona s'inserisce all'interno di una costante che esamineremo meglio nel prosieguo del presente lavoro.

Pur nella sua essenzialità - “los rahonaments ... son estats mols entre ells" - la nostra fonte ci permette di cogliere bene quali dovessero essere gli stati d'animo dei Sassaresi in quei giorni. E soprattuto anche quale pressione dovessero subire dagli eventi esterni che stavano preparandosi, in particolar modo legati alla ferma decisione del sovrano aragonese di proseguire con l'annessione al Regno di "Sardegna e Corsica" dei territori giudicali.

A causa dell'estrema incertezza della situazione "tot lo poble de Sàsser e les dones ab los infans als braços, cridant que abans se mengerien los fills que no consentrien que fossen de Catalans", si riunirono nella chiesa di S. Caterina all'interno della città dove si recarono anche il fratello del visconte di Narbona e il Maurellas ${ }^{10}$.

\footnotetext{
${ }^{10}$ La chiesa di S. Caterina citata nel nostro documento fu una delle cinque parrocchie in cui venne divisa la città di Sassari nel 1278 a causa dell'incremento del numero di abitazioni all'interno della cinta muraria, epoca in cui la città doveva avere una popolazione di circa dieci-
} 
Alla presenza dei due francesi avvenne il fatto più importante tra quelli narrati dall'informatore castigliano: i Sassaresi giurarono come loro signore, cioè come "jutge d'Arborea" Amerigo di Narbona, fratello del visconte, con alcune condizioni che servono a farcene comprendere meglio le modalità stesse. I Sardi, infatti, chiarirono subito che se Guglielmo II avesse deciso di tornare in Sardegna e di non osservare i patti stipulati con il re aragonese, tutta questa cerimonia di elezione sul trono giudicale di Amerigo era da considerarsi come non mai avvenuta ("no y haia res fet"). Nel caso in cui, però, Guglielmo II avesse persistito nel suo atteggiamento di accordo con il sovrano aragonese e nella sua intenzione di non ritornare nell'isola. i Sassaresi avrebbero riconosciuto Amerigo come loro legittimo giudice a partire dalla cerimonia di giuramento appena fatta ("axí, senyor, l'an jurat").

Emerge quindi per l'ennesima volta in maniera esplicita la determinata volontà dei Sardi, o almeno di una parte di essi, di rimanere fedeli alle istituzioni giudicali avvertite come più confacenti alle proprie esigenze di vita.

Risalta inoltre, tutto sommato, anche l'estremo tentativo fatto nei confronti di Guglielmo II, al quale sostanzialmente -almeno a nostro avviso- $i$ suoi sudditi con questa azione cercavano di forzare la mano perché non procedesse con la vendita del "Giudicato" al re d'Aragona. Eventualità quanto mai invisa ai Sassaresi, come emerge con chiarezza anche dalla nostra fonte.

Così come dalla scelta di Amerigo di Narbona come successore del fratello si evince che, pur essendo mutata sensibilmente la situazione, i Sardi non provavano un'avversione insanabile nei confronti della famiglia giudicale, che incarnava fisicamente in sé un patrimonio di sentimenti e valori politici, istituzionali e culturali nei quali una gran parte degli isolani

\footnotetext{
dodicimila abitanti. La chiesa di S. Catterina edificata prima del 1278, sita nell attuale piazza Azuni al lato del palazzo giudicale - poi palazzo del podestà quindi di nuovo pallazzo giudicale - era in comunicazione con questo tramite una tribuna. Andata in rovina dalla fine del XVIII secolo, essa venne abbattuta nel 1853 e la sua denominazione passoi alla chiesa gesuitical di Gesù e Maria. Data dunque l'ubicazione della chiesa e la sual contiguita con lédificio in cui sicuramente risiedevano sia Amerigo di Narbona che il Maurellas. risulta agevole comprendere perché i Sassaresi si riunirono li. Su questi edifici vedasi M. PORCu Gaias. Sassisari. Siontia architentonica e urbanistica dalle origini al '600. Nuoro, 1996. pp. 22. 50 e 94.
} 
si rispecchiava e che continuava a preferire a quelli importati dai CatalanoAragonesi".

Tornando adesso alla cerimonia di elezione. dopo essere stati giurati dal popolo sassarese anche Amerigo di Narbona e Pere de Montbrun toccando solenmemente con le proprie mani l'ostia consacrata procedettero al giuramento, non sappiamo se per effettiva convinzione o piuttosto per calmare gli animi dei Sardi decisamente agitati dopo le ultime notizie provenienti dalla penisola iberica.

Francamente, riteniamo difficile credere che $i$ due acconsentissero in maniera spontanea a sconfessare tutta la linea di condotta tenuta fino a quel momento da Guglielmo II con il re aragonese. Sembra essere più probabile che essi, vista soprattutto la situazione all'interno della città. abbiano quasi voluto rassicurare i Sassaresi. Ciò per diversi motivi: il primo dei quali è che essi non potevano ignorare di non avere la necessaria forza militare e politica per opporsi ad una decisione del legittimo signore d'Arborea, essendo ben consapevoli di trovarsi in Sardegna solo per farne le veci in sua assenza. Il secondo motivo è rappresentato dalla decisione dei due di porsi agli ordini dei Sassaresi ("a tot regiment et ordinació dels de Sàsser”) e di limitare i proprî spostamenti all'interno della città al castello

\footnotetext{
"All interno di questo discorso si collocanno alcuni documenti riguardanti sempre il Giudicato di Arborea e il suo signore Guglielmo II. redalti a Sassarti nel 1416. Si tratta di cinque documenti tutti indirizzati al giudice di Arhorea. al momento assente dall isola. e imviatigli dal fratello. dal signore di Maurellas. dal Podesta e dai consiglieri di Sassari e da Pietro de Feno che si definisce armentario de Logu. l'ufficiale che nel Giudicato dirigeval lesazione dei tributi - Iamministrazione dei beni fiscali. Cfr. F.C. CASul.A. La Sioria di Sardegha. cit. lemma 185. p. 172.

Nelle missive scritte dagli ufficiali giudicali al foro signore. aldili della retorica e dellenfasi sempre presenti in documenti simili. venne ribadito con molta insistenza il desiderio dei sudditi sardi di riavere in mezzo a loro il proprio signore. Eara una sorta di esigenzal fisica di poter vedere e quasi toccare il giudice. di poter verificare di persona che era ancora vivo e che si occupava degli interessi dei suoj sudditi. In questi documenti si ribadiva ripetutamente che la permanenza di Guglielmo II nel giudicato avrebbe rianimato i Sardi alla lotta comtro gli lonerici. Volendo fare un paragone. che solo a prima vista potrebbe sembrare improponibile. potremmo prendere in considerazione le quasi coeve lamentazioni presenti nei documenti catalano-aragonesi degli anni 20 del Quattrocento. originate dalla prolungata assenza dai territori iberici della Corona del re Alfonso V. impegnato nella diflicile concuista del Regno di Napoli. Assenza che poi com è noto divenne definitiva. essendosi trasferito il monarca a Napoli nel 1442 dove mori sedici anni dopo. Stesso discorso si puo lare. piü o meno per il medesimo intervallo cromologico, per if re di Francia Carlo VI. più volte costretto ad allontanarsi dalla capitale Parigi a causa delle continue occupazioni da parte delle truppe borgognone o armagnaceche.
} 
e negli spazi limitrofi fino alla chiesa di S. Caterina dove era stato prestato il giuramento ${ }^{12}$.

Il terzo elemento che ci spinge a formulare questa ipotesi è rappresentato dall'invio immediato a Narbona da parte di Amerigo e del Maurellas di un emissario - il francese che segretamente informò il gentiluomo castigliano degli eventi verificatisi a Sassari ("lo qual ve de part de n'Aymerich de Narbona e de Maurellas, he ve al vescomte de Narbona")- perché conferisse con il visconte e lo ragguagliasse della situazione che si era prodotta nella capitale giudicale.

A questo punto riteniamo opportuno fare una considerazione su quanto avvenne a Sassari in quei giorni pieni di avvenimenti convulsi. Essa si muove all'interno di un'ipotesi da noi avanzata in precedenti lavori, e riguarda i rapporti intercorsi tra il giudice Guglielmo II di Narbona e i suoi sudditi sardi. Rapporti imperniati su una mai sopita diffidenza degli isolani verso il loro nuovo signore, la quale sfociò in diversi tentativi di controllo dell'operato di quest'ultimo. E' questo il caso del consiglio di Oristanesi che secondo le intenzioni dei Sardi avrebbe dovuto affiancare Guglielmo II fin dal suo arrivo nell'isola, controllandone nel contempo l'operato soprattutto nei confronti dei Francesi ai quali non avrebbe dovuto concedere alcun titolo all'interno del regno giudicale ${ }^{13}$. Situazione che si ripresentò sostanzialmente invariata poco tempo dopo, quando, in seguito alla secessione di parte del territorio giudicale guidata da Leonardo Cubello, il visconte di Narbona venne nuovamente affiancato da un gruppo di cittadini di Sassari - divenuta la nuova capitale del regno arborense- senza il cui supporto la sua attività sarebbe stata impossibile, secondo quanto riportano in modo molto esplicito le fonti catalano-aragonesi $\mathrm{i}^{14}$.

Tornando ora al momento in cui venne effettuata l'elezione di Amerigo di Narbona a giudice di Arborea, i Sassaresi ottennero dal fratello del visconte e dal Maurellas non solo il giuramento di fedeltà alla linea

\footnotetext{
${ }^{12}$ Se si osserva una pianta della città di Sassari in età medioevale non si può fare a meno di notare che lo spazio lasciato al nuovo giudice e al suo tutore per i proprî spostamenti era veramente ridotto. Limitato ad una piccola porzione del centro della città aldilà del quale $\mathrm{i}$ francesi non avrebbero potuto recarsi, se non a rischio della loro incolumità personale.

${ }^{13} \mathrm{Cfr}$. L. Gallinari, Guglielmo III di Narbona, ultimo sovrano di Arborea, e la guerra dei Cent'Anni, in "Medioevo. Saggi e Rassegne", 18 (1993), p. 98.

14 "Era cierto que el vizconde no podía tratar ni obrar cosa alguna sino con consejo y voluntad de los de Sácer". Cfr. J. ZuRITA, Anales de la Corona de Aragón, ed. a cura di A. Canellas López, Zaragoza, 1974, lib. XI, vol. V, p. 234.
} 
politica di opposizione alla Corona d'Aragona da essi scelta, ma anche la sottomissione del nuovo giudice e del suo tutore alla volontà dei cittadini della capitale giudicale.

A questo proposito si può fare anche un'altra considerazione. Se da un lato traspare l'intenzione dei Sassaresi di mantenere il vincolo con la dinastia giudicale, fosse Guglielmo II o il fratello Amerigo, dall'altro risalta l'intenzione sempre più chiara di controllarne l'operato, atteggiamento che prima dell'insediamento sul trono oristanese di Guglielmo II non ha riscontro nella storia giudicale.

Sembra quasi di poter scorgere dietro questi avvenimenti una sorta di fase di trapasso istituzionale all'interno del "giudicato" arborense verso una sorta di monarchia parlamentare, in cui il consiglio dei maggiorenti dello stato indigeno sardo non solo era in grado di condizionare fattivamente l'operato del giudice ma addirittura giungeva a imporgli l'obbedienza alla propria linea politica in cambio del giuramento di fedeltà ${ }^{15}$. Detto questo, si può fare anche un'altra osservazione in merito alla fedeltà nei confronti della dinastia giudicale. Una fedeltà sui generis, probabilmente condizionata dalla situazione nella quale si trovava il regno indigeno che spinse il ceto dirigente di Sassari a non intraprendere una sollevazione aperta nei confronti del giudice Guglielmo II o dei suoi procuratori nell'isola, perché con ogni probabilità questa azione avrebbe potuto significare un'ulteriore spaccatura del regno arborense. In considerazione di ciò, fu scelta invece una politica di stretto e incondizionato controllo del sovrano, fosse il visconte di Narbona

\footnotetext{
${ }^{15} \mathrm{Per}$ il periodo precedente alla ascesa sul trono di Oristano di Guglielmo II abbiamo diversi riferimenti nelle fonti sarde e catalano-aragonesi all'operato della Corona de Logu (Parlamento dello Stato) - sorta di assemblea deliberativa formata dai rappresentanti dei liberi delle diverse curatorie del Regno di Arborea - al fianco dei giudici, soprattutto in concomitanza di stipule di accordi di pace, di trattati e di dichiarazioni di guerra. Occasioni in cui il suo intervento era in rappresentanza dell'intera comunità statuale. Non va infatti dimenticato che i Giudicati sardi erano Stati superindividuali, in cui era ben presente la distinzione tra quanto di pertinenza dello Stato e quanto invece spettante al giudice a titolo personale e privato. Sulla superindividualità dei regni giudicali sardi e sulle Coronas de Logu cfr. F.C. CASULA, La Terza via della Storia Pisa, 1997 , p. 88 . Sul controllo esercitato dai Parlamenti nei confronti dei sovrani medioevali vedasi invece $\mathbf{I b i d e m}$, p. 91

Anche tenendo ben presente quanto appena detto in merito alle competenze della Corona de Logu, ciò che si verificò a Sassari nell'estate del 1414 ha contorni molto diversi. Innanzitutto, nel nostro documento non si parla dei Sardi di tutto il Giudicato d'Arborea ma solo dei cittadini di Sassari. Per cui non sembra che sia possibile parlare stricto sensu di una Corona de Logu, almeno nell'accezione cui facevamo riferimento prima. In secondo luogo, in questa circostanza, il consiglio dei maggiorenti sassaresi non parve volersi limitare ad affiancare il legittimo sovrano nelle sue decisioni, ma giunse a pensare di subordinarne la elezione all'adesione al proprio programma politico.
} 
o suo fratello. Una sorta di compromesso che aveva il pregio di permettere di mantenere l'unità del regno arborense sotto il suo sovrano, le cui decisioni dovevano però essere prese insieme ai Sassaresi.

Questa nostra ipotesi sembra essere corroborata dal fatto che i Sardi, continuando a non fidarsi di Amerigo di Narbona e del signore di Maurellas, presero le loro misure di sicurezza emanando una grida in base alla quale $i$ Francesi e i Catalani che fossero stati trovati in giro nella città di notte sarebbero stati uccisi a colpi di virga, la tipica arma medioevale sarda.

I Sassaresi, in previsione di una lunga contrapposizione con le schiere iberiche - sempre secondo le informazioni fornite dalla spia castigliana- presero a rifornire di viveri e di altri beni necessari il solido castello che si trovava all'interno delal loro città. Oltre a ciò, essi fecero distruggere anche il ponte del castello e riattare il fossato della fortezza preparandosi così a sostenere anche un eventuale assedio, nel caso in cui le truppe catalano-aragonesi fossero riuscite a superare la cinta muraria che difendeva la città dall'esterno. I preparativi però non furono finalizzati solo alla difesa; i Sardi, infatti, avevano avviata la costruzione di una macchina da guerra ("giyn") che avevano deciso di posizionare in una piazza della città presso il castello così da aumentarne le capacità offensive ${ }^{16}$.

Sempre sul fronte offensivo, i Sardi e Amerigo di Narbona decisero di chiedere aiuti al di fuori dell'isola. Come sempre fino ad allora, la scelta ricadde sulla Repubblica Ligure. Per essere più precisi, da principio la richiesta venne inoltrata a Bonifacio, da dove erano già giunti nella capitale giudicale alcuni balestieri. in contemporanea, però, essendo ben consci che ciò non sarebbe bastato per affrontare l'offensiva iberica, Amerigo e i Sassaresi decisero di chiedere un sostegno alla loro azione militare anche a Genova, che già in precedenza aveva appoggiato Guglielmo II contro l'espansionismo aragonese. La città ligure avrebbe dovuto fornire due imbarcazioni e 300 uomini, attesi da un giorno all'altro al momento in cui partì da Porto Torres l'informatore francese. Non pago di quanto fatto, il nuovo giudice inviò una richiesta di aiuto anche a Marsiglia per riuscire ad

\footnotetext{
${ }^{16}$ Sul castello di Sassari vedasi F. FoIS, Castelli della Sardegna medioerale. Milano. 1992. pp. 213-219 e vedasi M. Porcu GaIas. Sassari, cit.. pp. 74-75, anche se in nessuna delle due ricostruzioni appaiono menzionati un fossato e un ponte. Da ciò che risulta dalla nostra fonte. sembra che il fossato non avesse più goduto di manutenzione da diverso tempo se i Sassaresi ritennero opportuno farvi alcuni lavori, segno questo che da parecchio la città non correva rischi di assedio.
} 
ottenere altre tre imbarcazioni (fustes) che avrebbero dovuto trasportare nell'isola rifornimenti in uomini e vettovaglie.

Da quanto finora riportato dall'informatore castigliano, sembra dunque emergere che effettivamente sia Amerigo di Narbona che i Sassaresi avessero deciso di provare a reggere l'urto delle truppe iberiche, rinforzandosi sia per terra che per mare. Continuiamo a ritenere che questo attivismo del giovane Narbona non andasse contro gli interessi di Guglielmo II. Crediamo piuttosto che egli abbia preferito assecondare $\mathrm{i}$ malumori provenienti dall'interno della città e salvaguardare così le prerogative del fratello, le quali altrimenti -almeno a nostro parere- sarebbero state definitivamente perdute in quanto i Sassaresi manifestarono con chiarezza la propria intenzione di proseguire comunque lo scontro armato con la Corona d'Aragona.

A prescindere da ciò, la spia castigliana aggiunse nel suo resoconto agli ufficiali regi di aver giurato a Amerigo di Narbona di portargli una risposta a tutte le richieste di cui egli era latore, e di essersi impegnato a trovare una imbarcazione a Marsiglia se non gli fosse stato possibile farlo a Narbona, dove si sarebbe dovuto recare per sollecitare aiuti.

Emerge ancora una volta un dato interessante. La comparsa di Marsiglia e della Provenza al lato dei Sardi e dei visconti di Narbona nella loro contrapposizione alla Corona d'Aragona. Era già avvenuto, anche se solo sul piano teorico, diversi decenni prima, nel $1377 / 1378$, quando il duca d'Angiò Luigi I, fratello del re di Francia Carlo V, aveva intavolato trattative diplomatiche con l'aliora giudice d'Arborea Ugone III per suggellare un'alleanza politico-militare tramite il matrimonio tra suo figlio e la figlia del sovrano oristanese Benedetta. I contatti erano ripresi circa vent'anni dopo, nonostante le mancate nozze, tra il giudice Guglielmo II di Narbona e il re di Napoli Luigi II d'Angiò, già promesso sposo della principessa oristanese, che ịnsieme a Jean Le Meingre offrì rifornimenti in vettovaglie e armi al visconte di Narbona nel 1412 contro la Corona d'Aragona ${ }^{17}$.

\footnotetext{
${ }^{17}$ Sui contatti tra le Case di Arborea e Angiò negli anni 1377/1378 fïalizzati all'organizzazione del matrimonio tra i rispettivi eredi vedasi R. CARTA RASPI. Ugone III d'Arborea e le due ambasciate di Luigi I d'Anjou, Cagliari, 1936. Sui sostegni forniti da Luigi II d'Angiò re di Napoli a Guglielmo II di Narbona tramite la persona del governatore francese di Genova. Boucicaut, ctir. Colección de documentos inéditos del Archivo General de la Corona de Aragón. ed. a cura di P. de Bofarull y Mascaró. Barcelona. 1848, tomo III, doc. $n^{\circ} 370$. p. 213. Con questo documento, inserito nei verbali parlamentari degli ultimi giorni di aprile 1412. la Deputazione generale di Catalogna informava i membri del Parlamento generale catalano di aver saputo dai consoli di Perpignano che "quatuor mille cesteriis frumenti et octo
} 
Questo ripetuto sostegno alla causa sarda giudicale secondo noi non deve però essere frainteso. Esso non va attribuito ad un particolare interesse della Casa d'Angiò nei confronti del Giudicato d'Arborea e delle sue vicissitudini, quanto piuttosto -almeno nei suoi primi momenti-al desiderio di dotare l'erede della potente famiglia francese di un trono dal quale continuare la contrapposizione sul piano militare e diplomatico alla Corona d'Aragona, con la quale lo scontro durava dall'epoca della guerra del Vespro.

Atteggiamento questo non eccessivamente dissimile da quello tenuto dalla Repubblica di Genova, che nel corso del Trecento e Quattrocento manifestò l'interesse di spalleggiare l'azione militare arborense contro la Corona d'Aragona, non tanto con lo scopo di poter poi realizzare conquiste territoriali dirette in Sardegna quanto invece con l'obiettivo di tenere impegnata nell'isola l'Unione iberica ed impedirle così di dedicarsi con maggiori mezzi alla penetrazione in Levante.

Un altro punto della nostra fonte merita di essere messo nel giusto rilievo. In quei giorni di concitati avvenimenti, in contemporanea a quella che trasportò l'informatore castigliano partì anche un'altra imbarcazione sempre diretta a Narbona che recava il nipote del signore di Maurellas, il quale - a dire del San Juan - rientrava in Francia per evitare che Guglielmo II venisse eliminato o a Narbona ("ací") o in Sardegna ("alà"). Altro elemento questo di estremo interesse perché potrebbe spingerci ad ipotizzare che Amerigo di Narbona e il signore di Maurellas temessero molto concretamente l'eventualità che i Sassaresi fossero giunti al punto di progettare l'eliminazione fisica di Guglilmo II, reo ai loro occhi di essersi accordato con il re aragonese, che sarebbe stato sostituito a questo punto in tutto e per tutto dal fratello Amerigo appena giurato giudice.

botes vini et ultra hoc sexaginta ballistarii" erano stati caricati da uomini di Narbona in favore del visconte, e che a Avignore il Boucicaut aveva ricevuto da Luigi d’Angiò re di Napoli la somma per arruolare ben 800 armati. Su tutto ciò cfr. anche L. GALlinARI, Sardegna, Genova e Aragona (1387-1416): relazioni politiche ed economiche, tesi dottorale, 1996. Questi contatti tra il re di Napoli e il visconte di Narbona furono fonte di preoccupazione per la regina Violante, moglie del defunto sovrano Giovanni I e suocera di Luigi II d'Angiò, la quale manifestò il timore che suo genero e suo nipote, tra i pretendenti al trono aragonese durante l'interregno, venissero esclusi dalla successione a causa di questi appoggi forniti a Guglielmo II. Cfr. L. GALlinARI, Riflessi della guerra tra Arborea e Aragona alla corte del re di Francia: nuove acquisizioni documentarie e prospettive di ricerca, in "Medioevo. Saggi e rassegne", 22 (1997), p. 160. 
Il definitivo deterioramento della situazione all'interno di Sassari e dei rapporti tra i Sardi giudicali e i Francesi è ulteriormente ribadito da un'altra affermazione contenuta nella nostra fonte. L'informatore castigliano espose agli ufficiali aragonesi che i Francesi, più che mai timorosi per la propria sopravvivenza, dubitavano di riuscire a tornare in patria e temevano di essere uccisi ("los francesos han dupte que los Sarts no.ls averguen"), mentre i Sardi paventavano che nel momento in cui si fosse proceduto al passaggio dei territorî giudicali in mano regia i Francesi e i Catalani, ormai d'accordo, avrebbero saccheggiato la città e il restante territorio ("que los Sarts an dupte que entre los Francesos et los Catalans no.ls meten a sachomano al liurar de la possessió"). Segno questo che, evidentemente, nonostante la progettata opposizione alla decisione del visconte e le dichiarate intenzioni bellicose, i Sardi prendevano nella adeguata considerazione che l'epilogo di quella vicenda fosse il ritorno all'obbedienza regia.

In conclusione della sua lunga relazione, l'ufficiale regio estensore della nostra fonte non azzardò ipotesi sul comportamento tenuto a Sassari da Amerigo di Narbona e dal signore di Maurellas, lasciando al sovrano in persona di decidere "si n'Aymerich de Narbona e Maurellas an fet açò ab cor de atendre ho per dupte de ramor". Anche se il semplice fatto che egli parlasse del timore di ramor, cioè di una sollevazione'dei Sardi che avrebbe spinto il fratello del visconte ad una simile decisione, fa pensare che tutta l'intera operazione di elezione di Amerigo di Narbona sul trono giudicale e la sua successiva accettazione apparisse alquanto strana per non dire insostenibile agli occhi degli stessi Catalani.

In questa direzione infatti si svolsero $\mathrm{i}$ successivi avvenimenti riguardanti gli ultimi anni di esistenza del Giudicato di Arborea. Legittimo signore del regno sardo rimase Guglielmo II, il quale continuò a trattare con i re aragonesi - prima Ferdinando I quindi Alfonso V- per la vendita dei suoi diritti sul regno arborense. Né d'altra parte, almeno fino ad oggi, sono stati rinvenuti documenti catalano-aragonesi indirizzati a Amerigo di Narbona nella sua veste di giudice d'Arborea, segno che per la Corona iberica il legittimo interlocutore rimase Guglielmo II.

Dalla documentazione posteriore finora in nostro possesso non abbiamo notizia di altri tentativi "insurrezionali" contro il visconte negli ultimi sei anni di vita del Giudicato di Arborea fino al 1420. Al contrario, più volte in altri documenti compaiono sia il visconte di Narbona che $\mathrm{i}$ rappresentanti della città di Sassari che trattavano con gli emissarî del 
sovrano aragonese le modalità di pagamento delle diverse rate in cui era stata frazionata la somma necessaria per l'acquisto del regno giudicale sardo ${ }^{18}$.

$\mathrm{Se}$, quindi, come abbiamo appena visto, da un punto di vista pratico l'elezione a giudice d'Arborea di Amerigo di Narbona non ebbe nessuna conseguenza, non altrettanto si può dire sul piano teorico da un punto di vista più strettamente giuridico e istituzionale.

Il tentativo effettuato dalla città di Sassari di controllare strettamente l'operato del giudice d'Arborea affondava le sue radici non solo nei tentativi già messi in atto negli anni immediatamente precedenti nei confronti di Guglielmo II. A parer nostro, si riallacciava anche ad una ancor più vecchia esperienza di autonomia cittadina che aveva trovato il suo momento di massima auge nel $1235 / 1236$ con la costituzione del Comune di Sassari ${ }^{19}$. Uno spirito di autogoverno più volte emerso con forza fin dall'inizio dell'inserimento della città all'interno. del Regno di Sardegna e Corsica, e che parve rinfocolarsi proprio in queste prime decadi del $\mathrm{XV}$ secolo, allorché, proprio sotto la guida di Guglielmo II di Narbona, la città divenne la nuova capitale del "Giudicato" di Arborea.

\footnotetext{
${ }^{18}$ Vedasi per esempio ACA, C. Cartas reales Alfonso V, cassa 19a, doc. 12. Interessantissimo documento datato 17 maggio 1417 dalla cui lettura risulta che in quella data a Sassari nel palazzo del visconte di Narbona alla presenza di Guglielmo.II, di alcuni cittadini di rilevo quali Pietro de Feno, che abbiano già incontrato in altri documenti col titolo di armentario de logu. Leonardo Cano e Giovanni Melone. oltre ad un ampia moltitudine di persone fu richiesto al giudice d'Arborea se intendesse mantener fede agli accordi presi con il defunto re d'Aragona Ferdinando I, che il figlio Alfonso V voleva mandare in esecuzione.

${ }^{19}$ Risulta estremamente attinente anche al periodo da noi esaminato nel presente documento la tesi sostenuta da A. CaStellaccio. Sassari medioevicile. I. Sassari. 1996, pp. 195-199. secondo la quale quella di Sassari era "una società forte consapevole della debolezza "storica" di un giudice di fatto [nel nostro caso. Amerigo di Narbonal ... pronta a cogliere o a cercare loccasione favorevole per modificare a proprio vantaggio gli equilibri politici e gli attuali rapporti di forza tra l'istituto giudicale e questa stessa società".
} 


\section{APPENDICE DOCUMENTARIA}

s.a., luglio, 12. Barcellona.

Un anonimo ufficiale catalano-aragonese, informato da una spia castigliana, Rodrigo de San Juan, della delicata situazione verificatasi a Sassari, capitale del "Giudicato" di Arborea, allorché vi si diffuse la notizia che il "giudice" Guglielmo II, visconte di Narbona, avev'a venduto i suoi diritti sovrani sullo Stato indigeno sardo al re d'Aragona Ferdinando I, espone dettagliatamente a quest'ultimo gli av'enimenti soffermandosi in particolar modo sulla nomina a nuovo sovrano d'Arborea effettuata dai Sassaresi nella persona di Amerigo di Narbona, fratello e luogotenente in Sardegna di Gugliemo II.

Archivo de la Corona de Aragón. Cancillería, Cartas reales Alfonso V. cassa 21 doc. $n^{\circ} 212$.

Molt alt e molt excel·lent príncep e senyor, ab humill reverència humilment notifich a vostra senyoria com hyr, que avien XI del present mes, ací aribà una fusta la qual venia de Serdenya, ço és dels parts de l'Alguer, e hés vinguda en VI jorns; en la qual fusta an venguts alsguns francesos de aquels que lo vescomte de Narbona havia jaquits a Sàcer, entre los quals ni ha un lo qual ve de part de n'Aymerich de Narbona e de Maurellas, he ve al vescomte de Narbona. Lo qual francès se's dexelat e molt secretejat ab un gentil hom ca(st)ellà qui solia estar ab lo vescomte, pensanse lo dit francès que encara hy stigués, lo qual ha nom Rodrigo de Sant Johan, e aquest Rodrigo és aquell qui tractava ab vostra senyoria e ab los Sarts. E lo dit francès hali dits les novelles seguents, he per avisar-vos, senyor, de les dites novitats yo les tramet a dita vostra se(nyor)ia en la forma que lo dit Rodrigo les ha comptadas al governador de Cathalunya he a mi. Et primerament diu que aprés que Barissony Cano, ço és lo sart qui era en companya del compte de Qui(rr)a, fonch a l'Alguer diu que los Sarts de Sàcer se aremoraren tots, e açò era a hora de miga nit. Per la qual ramor covench al germà del vescomte e a tots los altres francesos que's recullissen al castell, los qualls han estat III jorns que no son exits del castell. E stants los Sarts aremorats, dient que lo vescomte de Narbona los havia venits a vostra senyoria, e que ells no eren cans perqué deguessen ésser venits, e que abans se mengerien los fills que no consentrien que fossen de la casa d'Aragó. En açò 
Maurellas los preychà dient-los que no podie ésser que los (sic) vescomte agués finat ab vos, senyor, et en açò los Sards se abonencaren. Segui's aprés que un escuder del vescomte, lo qual lo vescomte trames de Saragossa en fora a Sàcer, al qual dien Rotxer, aribà a Sàsser en aquelles dies de la remor e comptà com vos, senyor, e lo vescomte ereu d'acord; de que si la primera remor fonch gran, molt major fonch aquesta altra. E en açò los Sarts vengueren e digueren a n'Aymerich de Narbona que si ell volia estar a regiment dels de Sàsser e que fessen ço que ells volguessen, que ells dependrien bens e personas e quant haven. En altra manera, ells sabien que s'avien a ffer. Los rahonaments, senyor, son estats mols entre ells. A la conclusió an finat en aquesta forma, ço és que tot lo poble de Sàsser e les dones ab los infans als braços, cridant que abans se mengerien los fills que no consentrien que fossen de Catalans, se son tots ajustats en una esgleya que és dins Sàsser, a la qual dien Santa Kathelina, e aquí és vengut lo germà del vescomte he Maurellas. E los de Sàsser han jurat per jutge d'Arborea al dit germà del vescomte ab condició, ço és, que si lo vescomte vol venir en Serdenya e que rompe los pachtes que a ffets ab vostra senyoria que no y haia res fet. Mas si lo vescomte no vol anar en Serdenya e vol tenir ço que ha promès a vostra senyoria, que en aquell cas ells volen e prenen ara per la vas lo dit Aymerich de Narbona per jutge. Axí, senyor, l'an jurat. E n'Aymerich de Narbona et Maurellas an jurat e fet sagrament sus la hostia sagrada, que no tendran neguns pachtes que lo vescomte haia fets ab vos, senyor, ans estaran a tot regiment et ordinació dels de Sàsser. Empero diu que per açò no està lo dit Aymerich que ell e los altres francesos no estiguen al castell e no n'ixen, sino fins a Santa Katerina. D'altra part, los Sarts se guayten molt bé, he an feta fer un crida que si Francesos ni Catalans van de nits que.ls averguen. Empero, senyor, diu que lo castell de Sàsser està bé fornit de viures he d'altres coses. An trencat los de castell lo pont, he han mes ha punt los valls. Los Sarts, axí mateix, fan adobar un giyn e han-lo assegut en una plaça pres del castell. Item, senyor, comte que n'Aymerich de Narbona e los Sarts de Sàsser han tramès a Boniffaci per balestes, e de fet son ja venguts a Sàsser. Item, més que han tramès per II navillis a Gènova, les quals deven portar CCC hòmens, e esperen si de jorn en jorn. Item, axí mateix, ha tramès a Masella per III fustes, les quals deven portar gent e viures. Item diu més que ell ha jurat a n'Aymerich de Narbona de tornar-li reposta de aquestes coses, e si a Narbona no trobe fusta que a Marsella ne deu trobar. Item, senyor, diu que quant ell partí de Sardenya ne partí una altra fusta on anava lo nabot de Maurellas, la qual anava a Narbona e aquest venia ací per ço que ho ací ho alà no arrassen lo vescomte. E diu més que los Sarts an dupte que entre los Francesos et los Catalans no'ls meten a sachomano al liurar de la possessió, e per semblant los Francesos han dupte que los Sarts no.ls averguen. Perqué, senyor, aquestes son les noves que lo dit francès compte. Si n'Aymerich de Narbona e Maurellas an fet açò ab cor de atendre ho per dupte de ramor, açò, senyor, avisera vostra senyoria. Perqué, senyor, de aquestes coses yo ab la present vull avisar vostra senyoria, per ço que en les 
cosses necessaries proveesque segons sa mercé sserà, e per aquesta rahó tramet a vostra senyoria lo present co(rr)eu menant a mi, molt alt et molt excel.lent príncep e senyor, vostra senyoria tot ço que sa mercé sser(à). Escrita a Barcelona a XII de juliol //

Al molt alt et molt excelent príncep et senyor rey d'Aragó et de Cicilia

\section{RÉSUMÉ}

A la lumière d'un nouveau document des Archives de la Couronne d'Aragon de Barcelona, cet article présente des nouveaux données sur la situation politique à l'interieur du Judicat ou royaume d'Arborée lorsque en 1414 à Sassari se répandit la nouvelle que le juge d'Arborea Guillaume II, vicomte de Narbonne, et le roi d'Aragon Ferdinan Ier étaient arrivés à un accord pour la vente des droits que le juge avait sur le Judicat sarde. En consequence de cet accort, la capitale judicale Sassari, en refusant la décision de Guillaume II, nomma nouveau juge d'Arborée Aymeric de Narbonne frere du vicomte, en lui prêtant serment.

\section{SUMMARY}

In the light of a new document that has been found in the Archivo de la Corona de Aragón in Barcelona, this article presents a new outline of the political situation in the interior of the "Giudicato" or Kingdom of Arborea while the news concerning the agreement between the "judge" of Arborea William II, viscount of Narbonne, and the king of Aragon, Ferdinand I, for the selling of the "judge" 's rights about the "Giudicato" spread in Sassari in 1414. In consequence of this agreement, Sassari, capital town of Arborea, refusing the William II's decision, elected new "judge" of Arborea Aymeric of Narbonne, viscount's brother, and took the oath. 